\title{
Philosophiques
}

\section{Le regard esthétique : Affect et violence, plaisir et catharsis}

\section{Christoph Menke}

Volume 23, numéro 1, printemps 1996

Critères esthétiques et métamorphoses du beau

URI : https://id.erudit.org/iderudit/027367ar

DOI : https://doi.org/10.7202/027367ar

Aller au sommaire du numéro

Éditeur(s)

Société de philosophie du Québec

ISSN

0316-2923 (imprimé)

1492-1391 (numérique)

Découvrir la revue

Citer cet article

Menke, C. (1996). Le regard esthétique : Affect et violence, plaisir et catharsis. Philosophiques, 23(1), 67-79. https://doi.org/10.7202/027367ar

\section{Résumé de l'article}

Un des problèmes centraux des discussions sur la relation entre éthique et esthétique tourne autour de la question de la violence. Celle-ci emprunte deux avenues dans le texte. La première traite d'aspects déterminés de la peinture de Francis Bacon, comme la façon dont l'art représente la violence. À ce propos, on formule la thèse que la provocation éthique de cette peinture ne repose pas tant sur le fait qu'elle représente (aussi) la violence, mais qu'elle engendre en sa présence un détachement esthétique, et même, un plaisir à l'occasion de cette présentation. La deuxième avenue proposée interroge l'effet éthique du plaisir esthétique en face de la violence. La théorie aristotélicienne de la catharsis est alors amenée comme modèle d'une « économie de la différence » qui permet d'élucider la relation entre plaisir esthétique et jugement éthique. 


\title{
IE REGARD ESTHÉEIOULE AFFECT EI VIOLENCE, PLAISIR ET CATHARSIS
}

\author{
PAR \\ Christoph Menke
}

\begin{abstract}
RÉSUMÉ : Un des problèmes centraux des discussions sur la relation entre éthique et esthétique tourne autour de la question de la violence. Celle-ci emprunte deux avenues dans le texte. La première traite d'aspects déterminés de la peinture de Francis Bacon, comme la façon dont l'art représente la violence. À ce propos, on formule la thèse que la provocation éthique de cette peinture ne repose pas tant sur le fait qu'elle représente (aussi) la violence, mais qu'elle engendre en sa présence un détachement esthétique, et même, un plaisir à l'occasion de cette présentation. La deuxième avenue proposée interroge l'effet éthique du plaisir esthétique en face de la violence. La théorie aristotélicienne de la catharsis est alors amenée comme modèle d'une "économie de la différence" qui permet d'élucider la relation entre plaisir esthétique et jugement éthique.
\end{abstract}

ABSTRACT : A central problem in the discussion about the relation between Ethic and Aesthetic is the question of violence. This question follows two paths in the text. The first one debates the determinated aspects in Francis Bacon painting, the way art represents violence. On that matter, we draw up the thesis that the ethical provocation in his painting don't stand (only) upon the representation of violence but also his tryptic creates an aesthetical indifference and even a pleasure. The second part questions the ethical effects of the aesthetical pleasure in front of violence. The aristotelien catharsis theory is therefore proposed as a model of an "economy of the difference "that allows the elucidation of the relation between aesthetical pleasure and ethical judgment.

Jusqu'à l'apparition de la nouvelle conception d'une " esthétique " à la fin du XVIII ${ }^{e}$ siècle, le lien étroit entre l'art et l'excitation intensifièe des affects ètait l'un des théorèmes centraux d'une théorie de l'art se fondant sur une doctrine des figures de la rhètorique et sur une détermination de l'effet des productions artistiques sur la sensibilitè. Or, comme l'esthétique depuis Kant, avec sa distinction entre les " beaux-arts " et les arts d'agrément, entre les arts purs et les arts appliqués, a détruit ce fondement rhétorique de l'art, ce lien ètroit entre l'art et l'intensification des affects a été rompu. Sa place 
est prise dans la nouvelle façon de voir de l'esthétique par la distinction stricte entre un plaisir immédiat, affectant la sensibilité, et un plaisir pur, réflexif - une distinction qui, sous une forme modifiée, détermine aussi les entreprises théoriques qui succéderont à l'esthétique kantienne, l'esthétique idéaliste avec sa conception de la vérité de l'art non moins que l'esthétique romantique avec sa conception de l'autonomie artistique. Depuis la seconde moitié du $\mathrm{XIX}^{\mathrm{e}}$ siècle, un nombre croissant d'auteurs ont voulu s'opposer à cette tradition qui s'est maintenue jusqu'à nos jours, tentant surtout, par le biais d'une réappropriation " physiologisante " de l'esthétique classique de l'effet (Bernays, Nietzsche), dirigée expressément contre la culture romantique et idéaliste de la réflexion, de rétablir la connexion entre l'art et l'affect par la dissolution de la distinction entre le plaisir des sens et un plaisir (purement) esthétique. Sous ce rapport, la recommandation de Nietzsche de mesurer l'effet du beau avec le dynamomètre plutôt qu'avec le goút réfléchissant du critique n'est que l'expression extrême de ces tentatives, lesquelles se sont d'ailleurs poursuivies, de manière différente, dans le pragmatisme américain $^{1}$ et dans l'esthétique française de l' intensitè " (Bataille, Klossowski).

Ayant dépeint cette situation théorique pour le moins riche en controverses, je voudrais proposer maintenant quelques réflexions prèliminaires sur le rapport entre théorie de l'art et théorie des affects. Elles se rapportent pourtant d'une manière ambivalente au dèbat qui vient d'ètre évoqué. Car, d'une part, elles s'inscrivent en faux contre la compréhension du plaisir esthétique comme effet de l'excitation artistique et de l'intensification des affects non-esthétiques. Avec Kant et le romantisme, et contre les diverses variantes, anciennes et modernes, d'une théorie rhétorique de l'art - centrée sur les affects - je comprends le plaisir esthétique plutôt comme un effet de la réflexion et de la liberté par rapport, justement, aux affects que déclenchent les objets de l'art. Je tenterai d'esquisser un rapport entre non pas l'excitation intensifiante, mais la réflexion distanciante des affects dans l'art et le plaisir esthétique qui lui correspond par le biais de quelques remarques sur un tryptique de Francis Bacon ( $1{ }^{\text {re }}$ partie).

D'autre part, toutefois, les réflexions qui suivent visent à montrer que la manière dont la théorie rhétorique de l'art formule les problèmes (et indique leur solution) ne perd rien de sa pertinence même lorsqu'on s'oppose à sa détermination de l'art comme intensification des affects. Cela touche en particulier au problème " politique "de la violence de (dans) la production artistique. Ce problème est "politique "parce qu'avec la question de la violence de (dans) l'œuvre d'art se pose du mème coup celle de la possibilité d'une communauté vivant "en harmonie " et du risque qu'elle court d'en ètre minée. C'est alors que la théorie rhétorique de l'art touche à ce problème de la violence par un double biais: la question se pose dans la mesure où la violence est à la fois objet et propriété des

1. Là-dessus, voir à présent les études impressionnantes de R. Shustermann, Kunst Leben. Die Asthetik des Pragmatismus, Fisher Verlag, Francfort, 1994 (L'art à l'état vif. La pensée pragmatiste et l'esthétique populaire, Paris, Minuit, 1991). 
affects produits par l'œuvre. Par la brève description du tryptique de Bacon il sera montré qu'en abandonnant la théorie rhétorique au profit de la théorie réflexive de l'art, le problème de la violence se pose certes autrement, mais de façon non moins pressante. C'est pourquoi j'esquisserai dans la seconde partie la solution au problème de la violence de (dans) l'œuvre d'art que la théorie rhétorique a trouvée dans le concept aristotélicien de catharsis $\left(2^{c}\right.$ partie), pour ensuite examiner la possibilité de reformuler cette solution eu égard à la problématique quelque peu différente de la violence dans la théorie réflexive de l'art ( $3^{e}$ partie).

\section{Bacon}

Au centre des trois tableaux formant le Tryptique d'août $1972^{2} \mathrm{de}$ Bacon se trouve à chaque fois une figure que l'on peut reconnaitre comme ètant, de façon plus ou moins rudimentaire, encore humaine. Ce qui frappe dans ces figures, ce sont les traces d'une double dissolution des limites du corps, tant vers l'extérieur que vers l'intérieur. Ces traces qui, comme celles que l'on peut voir dans les photographies policières prises sur les lieux du crime, mettent en mouvement l'imagination romanesque de l'observateur, sont en premier lieu ces taches de couleur qui paraissent représenter des flaques de liquides corporels sortant des figures, dans lesquelles elles semblent mème se dissoudre. Ces traces sont aussi, en second lieu, des atteintes déformantes aux contours nettement dessinés et à la corporalité des figures - des trous, des discontinuités - , et aussi et surtout aux visages qui ont été comme écrasés et déplacés.

Mais, plus que tout, c'est la séquence du tryptique qui suggère une lecture narrative de ces atteintes au corps en tant que signes d'un acte de violence passé. Les tableaux du tryptique apparaissent alors comme les stations d'un chemin de la douleur que l'observateur devine à partir des traces visibles qu'il a laissées sur le corps de la figure. Une telle description du contenu de cette œuvre, avec ses affects moraux de l'horreur et de la pitié, ne parvient pourtant pas, et de loin, à traduire l'impression de violence qu'elle suscite. Dans le Tryptique d'août 1972 comme dans plusieurs tableaux de Bacon, ce qui est décisif, ce n'est pas tant qu'il montre la seule déformation violente des corps, mais en outre et plutôt l'exposition de ces corps déformés et leur mise en pâture à une observation voyeuriste, laquelle transforme le sentiment d'horreur devant la déformation des corps en un sentiment de plaisir, le plaisir de regarder. C'est que les panneaux de gauche et de droite du tryptique de Bacon déplacent sur une scène ou sur une rampe le corps déformé et en état de dissolution. L'objet du tableau devient ainsi réflexif : il n'y va pas seulement de ce qui s'y fait voir, mais de ce qu'il soit vu et de la façon dont il est vu. En effet, le regard que montre le tableau de Bacon est un regard porté de loin : de la distance infranchissable qui sépare les spectateurs de ce qui est vu sur la scène. Le tableau montre un

2. Chaque tableau mesure 198 × 147,5 cm ; Tate Gallery, Londres. Une première version de cette description de l'œuvre de Bacon se trouve dans Ch. Menke, "Schrecklich schön. Bemerkungen zu Marcuse und Koppe, im Blick auf Bacon *, in Institut für Sozialforschung (éd.), Kritik und Utopie im Werk von Herbert Marcuse, Suhrkamp, Francfort 1992, p. 262-271. 
regard qui théâtralise et irréalise la violence présentée, éloigne la violence et de ce fait la redouble : dans l'indifférence devant la violence, voire dans le plaisir pris dans sa présentation. Dans le tableau de Bacon, le regard plein de pitié et d'horreur porté sur la violence qui a laissé ses marques sur un ètre humain est courtcircuité par le regard appréciatif porté sur cet objet de curiosité. Le tableau de Bacon est le lieu du croisement de ces deux regards. Ce lieu n'est cependant pas celui d'une rencontre, où les deux regards se rejoindraient. Il s'agit plutôt du lieu d'une "rencontre "au sens agonal du terme : de leur conflit. Le tableau est le champ de bataille des regards.

Il ne faudrait pas comprendre que le tableau de Bacon ne fait que présenter le regard accompagné de plaisir porté sur la figure déformée, qui courtcircuite le regard de pitié et d'horreur porté sur la violence, ou même que par là il le dénoncerait. Il y a plutôt une complicité souterraine entre son tableau et ce regard. Car c'est justement cette sorte de regard voyeuriste, distanciant, que le tableau de Bacon suscite chez le spectateur, puisqu'il s'agit du regard par lequel il se produit comme tableau. Dans la thematisation reflexive du regard distanciant porté sur la figure déformée, le tableau de Bacon accomplit aussi une autoréflexion du regard grâce auquel il s'est constitué. De façon générale, la thématisation autoréflexive de situations du regard et de l'observation, de la (l'auto-) présentation et représentation fait partie des motifs fondamentaux de la peinture baconienne : par exemple, dans les accessoires et les motifs du miroir, de la caméra, du journal et de l'écriture, de la photo ou de l'image, de la figure observante, de la lampe qui éclaire et qui rend possible la vue, ou encore de la fenetre et de la porte par lesquelles quelqu'un peut regarder. Ici toutefois, le tableau de Bacon est autoréflexif surtout dans sa présentation et sa mise en scène d'un regard qui se distancie des déformations d'un corps, voire qui s'en repait. C'est pourquoi ce regard dans le tableau n'est autre que le sien, lequel produit le tableau et le met en scène ; il s'agit du regard esthétique. Même la déformation de la figure revêt pour ce regard une autre apparence : la mutilation violente d'un corps humain lui apparaît comme élément d'une mise en scène; on peut même dire qu'il s'agit pour lui d'un acte d'une expérience esthétique. C'est pourquoi la pratique esthétique est déjà elle-même, et pas seulement son contenu, une déformation violente. Sous ce rapport, Leiris a parlé de la peinture baconienne comme d'une "agression " et d'un "acte de violence ", et Bacon lui-mème a parlé d'un fait "cru " et d'un "accident ": ce qui est arrivé aux corps qu'il présente comme un " accident " et qui a laissé en eux ses traces n'est rien d'autre que le fait d'être présentès ; et ce qui pouvait arriver de pire aux figures de Bacon, c'est qu'elles soient devenues ses figures.

L'art de Bacon interdit ainsi à son spectateur de se donner bonne conscience devant la mise en scène artistique de la violence en prétendant qu'elle est démentie et dénoncée dans cette mise en scène. La réponse bien connue de Picasso à la question d'un officier allemand lors de la contemplation de "Guernica ", qui voulait savoir qui avait fait cela, était certes déjà politiquement correcte du temps de Picasso, mais trop facile d'un point de vue esthétique. Car l'artiste aussi a "fait quelque chose ": non pas "Guernica ", certes, la des- 
truction barbare d'un village, mais il a fait de "Guernica I", l'événement historique, "Guernica 2 ", un tableau d'une composition artistique achevée, hautement articulé, esthètiquement réussi et qui pour cette raison nous plait (et qui plus tard est devenu "Guernica 3 ": un événement médiatique, un poster dans d'innombrables communautés d'habitation, du kitsch politique). Et cela vaut aussi pour le tableau de Bacon : parce qu'il représente la déformation violente des corps et qu'il présente en méme temps sa propre mise en scène esthétique, il donne à cette violence, comme le dit John Berger dans un rapprochement polémique des figures de Bacon avec celles de Walt Disney, une "forme absolue ${ }^{3}$. Berger se réfère avec raison à la complicité que l'art de Bacon établit entre ce regard de la jouissance détachée et la déformation violente. Mais il manque au diagnostic de Berger l'intuition de ce que l'art de Bacon acquiert grâce à cette complicité : une beauté d'un genre tout nouveau et particulier. Il acquiert cette beauté justement dans le détachement du regard qu'il jette sur ses figures. Bacon dit à propos de cette bouche qui, dans sa célèbre série de tableaux de papes, crie son horreur: "J'aime pour ainsi dire l'étincellement et la couleur qui sortent de la bouche, et j'ai toujours en quelque sorte espéré pouvoir peindre la bouche [donc: la bouche déformée par un cri d'horreur] comme Monet a peint un coucher de soleil ${ }^{4}$ ". Ainsi cependant, dans l'étincellement de sa couleur, la bouche poussant un cri ne se laisse peindre que si le regard esthétique l'isole de tous les contextes dans lesquels nous la percevons par sympathie comme le geste expressif d'un sujet qui souffre. Le regard esthétique dépossède ainsi le sujet de ses modes d'expression qui lui semblent les plus immédiats et intimes : ce qui déclenche chez l'observateur les réactions morales et affectives les plus intenses - l'expression de la souffrance dans des cris ou dans des gestes; les traces de la violence dans la destruction et la déformation - est justement ce qui apparait au regard esthétique comme ce qui n'est plus, selon les propres mots de Bacon, que "real " ou "factual ": pur ètre-là, sans rapport avec rien dautre. Cette dissolution de l'expressif et de l'affectif dans la pure facticité se laisse mème retracer jusque dans la matérialité de ses tableaux, dans le choix de la couleur et la manière de son application. Car ce qui la détermine est le contraste entre les acryliques mates avec lesquelles Bacon peint le sol et les huiles brillantes (qu'il mélange en outre avec du sable, des filaments et des cheveux et qu'il structure spatialement à l'aide d'empreintes de tissus) par lesquelles il fait ressortir certains éléments des figures qui commencent ainsi à briller et - selon le mot de Bacon - à "étinceler ". La peinture de Bacon obtient justement des objets ses réactions morales et affectives les plus fortes en mēme temps que la plus haute intensité de son effet esthétique au moyen d'une telle réduction à leur facticité pure, ne laissant aucune place à une interprétation et à un jugement. Les éléments que Bacon fait ressortir des figures au moyen de leur matérialité "étincelante " sont précisément ces traits dans les figures baconiennes qui apparaissent comme des effets de la violence au double sens du mot : aussi bien

3. J. Berger, "Francis Bacon and Walt Disney ", in (du même auteur), About Looking. Pantheon, New York 1980, p. 111-118.

4. D. Sylvester, Gespräche mit Francis Bacon, Prestel, Munich 1982, p. 52. 
de cette violence dont la peinture de Bacon présente les effets que de celle dont elle est elle-même l'effet. Le tableau de Bacon doit à cette violence intensifiée par redoublement les moments de sa plus haute beauté et son plaisir esthétique.

Avant que je n'aborde la question de la signification de cette œuvre pour le problème "politique "touchant la violence de (dans) l'œuvre d'art, j'aimerais résumer brièvement l'aspect de l'esquisse précédente le plus pertinent pour celui-ci. Il s'agit de l'imbrication complexe de la violence et de la réussite artistique dans la peinture baconienne. Nous avons d'abord vu que la force et l'intensité des affects surtout moraux devant la violence qui domine entièrement le tableau de Bacon ne se laissent comprendre que si la violence n'agit pas seulement comme événement représenté, mais dans l'événement même de la présentation: dans le regard se détachant de son objet pour en faire un objet de curiosité, que l'on prend plaisir à regarder, regard que le tableau de Bacon jette sur la déformation violente des corps et qui l'affirme comme son acte propre. Le regard esthétique répète ainsi et intensifie par là la violence représentée. On lui doit cependant aussi le plaisir esthétique pris à ces tableaux. Car le caractère violent du regard esthétique consiste justement dans l'isolement des traits des corps humains déformés qui sont le plus immédiatement associés - comme effets ou comme expressions - à la violence qui leur a été faite et qui, de ce fait, sont les objets des réactions morales et affectives les plus intenses, dans leur sortie complète hors de ce contexte pour, dans l'étincellement de sa couleur, en faire l'objet d'une pure contemplation esthétique. Dans ces moments du plaisir esthétłque, la répétition esthétique de la violence se fait donc telle que nous ne la percevons plus comme violence. Le plaisir esthètique que l'on doit à la violence contredit du mème coup les affects de la pitié et de l'horreur dans lesquels la contemplation du tableau révèle sa violence par un jugement moral. À l'inverse, poser la violence comme fondement du plaisir esthétique implique qu'on ne la tienne plus pour un phénomène moral, ou qu'on ne la perçoive et ne la juge plus à partir d'une perspective morale. La répétition esthétique de la violence implique donc rien de moins qu'une émancipation du sujet par rapport à la perspective morale. Le plaisir esthétique et la beauté signifient la liberté : non pas, comme Schiller l'entendait, une liberté morale, mais, comme Nietzsche l'a compris, une liberté esthétique, à savoir une liberté par rapport à la perspective morale elle-même.

\section{Catharsis}

Avec la connexion entre violence et réussite artistique dont la peinture de Bacon nous offre l'exemple, nous avons caractérisé du mème coup l'état du problème "politique "qui se trouve réfléchi dans cette peinture. Ce problème politique, qui se pose dans la peinture baconienne, peut ètre circonscrit par les conséquences politiques de l'art découlant de ce qui se passe sur le plan esthétique dans l'art, conclusion qui peut être tirée à partir de l'esquisse précédente sur Bacon. D'après ce raisonnement, ce qui s'accomplit (esthétiquement) dans l'art et l'effet (politique) causé par l'art, c'est la même chose: une affirmation de la violence et la dissolution des normes morales 
sur lesquelles on se fonde pour la condamner. Or, on peut faire valoir l'argument selon lequel si l'art ne doit sa réussite et son plaisir qu'au redoublement de la violence par l'indifférence à l'égard de la perspective morale, lart a alors aussi pour effet un redoublement politique de la violence par l'indifférence à l'égard de la perspective morale.

Cet argument parait simple et convaincant. J'aimerais toutefois montrer dans ce qui suit qu'il est trop simple (et pourquoi). Il est trop simple parce qu'il présuppose une fausse compréhension du rapport entre art et politique. Ce modèle est déterminé par une logique de l'identité ou, plus précisément, par la supposition d'une traductibilité immédiate d'expériences esthétiques en expériences politiques : ce qui est pratiqué en art signifierait donc la même chose pour la politique, comme si cela était immédiatement pratiqué en politique. Suivant ce modèle, il y aurait convertibilité entre ce qui se passe en art et la pratique politique, une convertibilité qui est censée ètre illimitée tant des points de vue quantitatif que qualitatif. Cependant, il y a un prix à payer pour cette image homogénéisante : on perd de vue les frontières existant de fait entre les différents domaines, au passage desquelles les significations font l'objet d'un échange obligatoire ${ }^{5}$. Certes, cette frontière entre la politique et l'art ne sépare pas les uns des autres leurs contenus et leurs expériences, mais elle exige pour leur passage dans l'autre domaine le prix de leur transformation. S'il y a donc une économie englobant la politique et l'art, alors, c'est sous la condition que l'on respecte ces frontières. Contrairement à la fausse supposition de leur identité, il prévaut dans le rapport entre art et politique une économie de la différence.

J'aimerais maintenant formuler de manière plus précise et concrète ces remarques passablement générales et abstraites en me référant au premier débat connu sur la signification de l'intensification de la violence (ou de sa présentation) en art pour le problème des mesures à prendre afin d'empècher la violence (ou son emploi) en politique. Il s'agit du débat entre Platon et Aristote sur la tragédie et sur le rôle qu'elle joue pour une bonne vie (en commun) dans la Cité. Il faut dire tout de suite que ce problème "politique " de la tragédie pour Platon et Aristote se distingue-comme nous le verrons bientôt - sous un rapport fondamental de celui que nous venons d'observer chez Bacon : Platon et Aristote perçoivent le problème politique de la tragédie non dans la libération esthétique des affects moraux par rapport à la violence, mais dans les effets pour la Polis d'une excitation et d'une intensification de la violence des affects moraux. Pourtant, Platon et Aristote proposent deux modèles du rapport entre tragédie et Polis dont on montrera qu'ils peuvent tout

5. L' échange obligatoire * (Zwangsumtausch) était naguère une mesure en vigueur dans tous les pays du bloc soviétique forçant les visiteurs étrangers à écharıger une certaine somme en devises * fortes * contre un montant en monnaie nationale au passage de la frontière. Par exemple, au passage de la frontière est-allemande, on exigeait du visiteur occidental qu'il échange 13 Deutsche Mark contre 13 Ost-Mark (nom de la monnaie de la République démocratique d'Allemagne), alors que sur les marchés libres en Occident, et davantage encore sur les marchés noirs en R.D.A. même, l'Ost-Mark ne valait qu'une fraction du DM, soit de un sixième à un dixième. [Note du traducteur]. 
de mēme servir à cerner le problème politique qui se pose en rapport avec le tryptique de Bacon.

Mais d'abord, je rappellerai très brièvement la description de l'effet affectif de la tragédie et de la problématique politique qui lui est liée, description élaborée par Aristote à la suite d'une formulation antérieure de la question par Gorgias et Platon. Au centre de cette détermination se trouve ce qu'Aristote appelle le "mythe "de la tragédie : soit l' assemblage "poétique "des événements "(Poétique, $1450 \mathrm{a}^{6}{ }^{6}$ conformément à l'action tragique avec son basculement caractéristique de la vie d'une personne ordinaire (du point de vue de la vertu) d'un état de bonheur dans le malheur. C'est cette action dont la présentation dramatique - dans laquelle "toutes les figures entrent en scène comme agissantes et en pleine activité "(1448a) qui provoque les affects tragiques, eléos et phòbos, que l'on traduit maintenant, sous l'influence surtout de Schadewalt, par " lamentation " (Jammer) et "frisson d'horreur " (Schauder) ${ }^{7}$. Les spectateurs de la tragédie déplorent le malheur d'un ètre humain, parce qu'il s'est abattu sur lui sans qu'il ne l'ait mérité, et cela les fait frissonner d'horreur parce qu'il est leur "semblable". Le débat entre Platon et Aristote porte donc sur cette question : s'il est bon (pour la Polis) que la tragédie suscite ces affects dans cette mesure sans pareille, voire dans cette démesure.

Comme on sait, Platon répond par la négative et Aristote par l'affirmative à cette question ; tandis que Platon soutient que l'excitation des affects par la tragédie a un effet délétère sur les vertus qui sont essentielles pour la Polis, Aristote attribue à l'excitation des affects par la tragédie un effet "purificateur " par lequel la Polis est renforcée. Eu égard à l'argumentation déployée par Platon au Livre $X$ de la République, deux aspects dans ce débat se laissent distinguer. Le premier argument de Platon contre la tragédie renvoie au portrait du monde éthique qu'elle dessine. Suivant cet argument, le monde èthique est falsifié dans la tragédie d'une manière telle que cela a des implications pratiques graves : puisque la tragédie montre comment des personnes qui ne sont pas sans vertu, voire sont vertueuses, tombent dans le malheur et la souffrance, elle affirme implicitement une différence entre bonheur et vertu, différence qui non seulement ne tient pas en vérité - c'est-à-dire si l'on a correctement compris ce en quoi consiste le bonheur véritable --, mais qui, en outre, si l'on y croit, amène les gens à poursuivre un faux bonheur au détriment de la vertu. Ainsi, tandis que Platon déclare comme étant nécessairement fausse, parce que copie seulement de l'apparence de la vérité, l'impression de pluralité et de fatalité, de "fragilité "(Martha Nussbaum) que la tragédie donne du monde éthique, Aristote, de son côté, conteste jusqu'à la possibilité de juger en termes de vérité ou de fausseté de la révélation du monde qui s'accomplit dans les affects

6. Je me suis servi des traductions suivantes : la Poétique d'Aristote par M. Fuhrmann, Reclam, Stuttgart, 1982; la Politique d'Aristote par E. Rolfes, Meiner, Hambourg 1982, et la République de Platon par F. Schleiermacher. Insel, Francfort, 1991.

7. La lecture traditionnelle de ces deux termes est : " pitié * et " crainte ". Cf. l'édition de J. Hardy, lère èd., Soc. d'édition * les Belles Lettres *, Paris 1932, p. 37. [Note du traducteur]. 
tragiques : l'apparition du monde èthique dans la tragédie s'accomplit "suivant la vraisemblance " (Poétique, 1451 b).

Ce premier niveau du débat entre Platon et Aristote a trait au contenu cognitif et à la fiabilité épistémique des affects tragiques. Pour notre propos, c'est toutefois le deuxième niveau du débat qui est décisif, lequel a trait à la violence, à l'excitation et à l'intensification de ces affects par la tragédie et à son effet sur la Polis. Quoique le jugement de cet effet soit chez l'un à l'opposé de ce qu'il est chez l'autre, ils partagent tous deux une présupposition essentielle, à savoir que l'excitation de l'affect par la tragédie présente un problème pour les vertus dans la Polis dans la mesure où il n'est pas possible de les exercer en mème temps que les affects tragiques sont suscités ; il s'agit là de la négativité fondamentale de l'excitation des affects tragiques par rapport aux vertus. Mais à partir de ce point de départ commun, Platon et Aristote arrivent à leurs évaluations diamétralement opposées de la tragédie. Ainsi, Platon est convaincu que l'excitation intensive des affects, qui a lieu dans la tragédie, renforce, de manière générale, ces affects et donc aussi dans la Polis. Par conséquent, l'affaiblissement de la vertu, qui est liée à une excitation démesurée des affects, n'est pas limitée au seul domaine de la tragédie, mais s'étend aussi au domaine de la Polis : la poèsie (moins la "partie "qui "produit des hymnes aux dieux et des chants faisant l'éloge d'hommes excellents"; République, 607 a) a pour effet "que soient négligées la justice et les autres vertus " (608 b) ; elle fait que " plaisir et déplaisir gouvernent dans l'État, au lieu des lois et des pensées raisonnables tenues à chaque fois dans la communauté pour indiquer ce qu'il y a de meilleur " $(607$ a). Car les effets de la poésie consistent en un renforcement des pouvoirs de l'affect, pouvoirs "éloignés de la Raison" (603 a), et elle y procède avec une violence si irrésistible que même ceux qui sont "bien intentionnés " ne peuvent y échapper. Il est clair que Platon conçoit le rapport entre la tragédie et la Polis suivant la logique de l'identité mentionnée plus haut : l'excitation des affects dans la tragédie est la mème que dans la Polis, et cela signifie surtout la mème chose, à savoir un affaiblissement des vertus sur lesquelles repose la Polis.

Or c'est ce raisonnement conforme à la construction identitaire du rapport entre art et politique dont la logique est remise en question par Aristote en se référant à l'effet cathartique de la tragédie. Dans la Poétique, Aristote décrit (et justifie) la tragédie en disant de celle-ci qu'elle " suscite lamentations et frissons d'horreur et accomplit par là une purification de tels états d'excitation "(1449 b). Par cette thèse, Aristote confirme, dans un premier temps, la négativité de l'excitation affective soulignée par Platon, en tant qu'elle affaiblit la vertu. Car s'il n'y avait aucun problème pour la Polis et ses vertus dans l'excitation des affects tragiques, l'indication selon laquelle cette excitation s'accompagne d'une purification et n'est donc pas nocive (comme Aristote l'écrit dans le Livre VII de la Politique) serait superflue. Mais avant tout, avec le théorème de la purification, Aristote indique la voie d'une toute nouvelle solution au problème. Aristote dit en fait, avec son modèle de la catharsis, rien d'autre que le problème est déjà aussi sa solution. Car la solution du problème que pose l'excitation des affects tragiques pour les vertus de la Polis tient justement au fait que cette excitation a lieu dans la tragédie. Et susciter les affects 
dans la tragédie signifie non pas justement les susciter aussi dans la Polis, mais de les désactiver et donc de ne pas les susciter dans la Polis. Le modèle aristotélicien de la catharsis ne rencontre donc pas l'expulsion platonicienne de la tragédie hors de la Polis en contredisant sa description de la tragédie comme d'un lieu de la remise en question de la Polis et de l'affaiblissement de sa vertu par une excitation démesurée des affects, mais en soulignant l'effet "homéopathique "- le mot est d'Aristote (Politique, 1341 b) positif d'un tel affaiblissement de la vertu pour la vertu. La tragédie pratique ce qui est étrange pour la Polis, la remettant même en question - et c'est ce qui est justement bon pour la Polis. Tandis que Platon ne pouvait se représenter la connexion entre tragédie et Polis que comme leur identité, Aristote propose dans la théorie de la catharsis le modèle d'une économie commune issue de la différence : la tragédie et la Polis collaborent parce que et pour qu'elles se limitent et se distinguent mutuellement, allant mème à contrecourant l'une de l'autre.

\section{Délestement ${ }^{8}$ et connaissance}

Revenons maintenant à l'art de Bacon et demandons quelles sont les conséquences qui sont à tirer du modèle de la catharsis avec son économie de la différence pour la question de l'effet politique de cet art. Sous ce rapport, il faut d'abord prendre en considération la différence fondamentale que présente cette question par rapport au problème de la tragédie, différence que j'ai déjà évoquée plus haut. Car pour ce qui est de la tragédie, le concept aristotélicien de la catharsis devait servir à répondre à deux questions à la fois : à la question du plaisir spécifique que la tragédie éveille chez le spectateur, et la question des retombées bénéfiques de la tragédie pour la Polis. Aristote comprend les deux - le plaisir (esthétique) et les retombées (politiques) - comme la même purgation des affects tragiques suscités : la purification, qui est liee à l'excitation des affects, explique - suivant toujours une formulation que l'on trouve au Livre VIII de la Politique - pourquoi l'excitation et l'intensification des affects tragiques est également "bénéfique "-c'est-à-dire plaisante pour les spectateurs - et " non nocive " - c'est-à-dire utile pour la Polis. En ce qui concerne l'art de Bacon, toutefois, dont j'ai tenté plus haut d'esquisser l'expérience, cette connexion immédiate entre plaisir esthétique et utilité politique dans le concept aristotélicien de catharsis ne peut être maintenue. Car le plaisir esthétique pris à la contemplation de l'art baconien est centré sur la beauté de sa présentation en conflit avec la violence et les affects " tragiques " qui y sont présentés, qu'il suscite. C'est pourquoi le plaisir esthétique signifie dans ce cas une libération par rapport aux affects tragiques, laquelle mine du même coup la perspective morale de façon

8. Entlastung signifie "enlèvement d'un poids, d'une charge *, donc littéralement : " décharge *. Mais comme il ressort de ce qui suit, l'idée est celle d'un allègement momentané de la pression morale, sociale, par l'art. Le terme que nous avons choisi pour traduire cette idée contient aussi l'idée d'un allègement et est du reste de la même racine que le mot allemand Entlastung. Nous avons préféré le néologisme " délestement " à " délestage ", dont l'emploi est trop technique, ainsi qu'à " délassement * qui ne traduit pas toutes les connotations du terme allemand. [Note du traducteur]. 
générale : le plaisir esthétique pris à la contemplation de la beauté est ici le médium d'une liberté esthétique par rapport à la perspective morale. Mais cela signifie aussi, du mème coup, que le plaisir esthétique dans le cas de Bacon ne peut pas aussi déjà, comme Aristote l'avait affirmé pour le cas de la tragédie, présenter une solution du problème politique, mais signifie au contraire son aggravement à l'extrème. Tandis qu'Aristote voit dans l'excitation démesurée des affects tragiques par la présentation intensifiée de la violence dans la tragédie le problème menaçant la Polis et sa vertu, et dans le plaisir esthétique, en tant que plaisir pris à l'effet purificateur et calmant de cette excitation, la solution de ce problème politique, donc, contrairement à cette identification du plaisir esthétique à l'utilité politique chez Aristote, le problème que pose un art comme la peinture baconienne, c'est le fait justement qu'il en pose un pour la vie en commun et ses fondements moraux en raison mème du plaisir esthétique qu'il suscite. Car le plaisir esthétique est lié de manière constitutive dans l'art baconien à (la répétition artistique de) la violence et est ainsi le médium d'une libération par rapport à la perspective morale à partir de laquelle nous la percevons, la ressentons et la jugeons comme violence.

Malgré cette différence essentielle dans la façon de poser le problème, l'idée fondamentale de la solution qu'Aristote a trouvée dans le concept de catharsis pour la tragédie se laisse aussi reformuler pour l'art baconien. Car le noyau du modèle de la catharsis consiste en une économie de la différence. En effet, la tragédie a un effet bénéfique pour la Polis justement en tant que lieu de l'activité de ce qui est nocif pour la Polis. Une telle logique de l'inversion est valable également pour l'art baconien - mème si le " nuisible " qui sévit en lui ne consiste pas en une excitation démesurée des affects tragiques, mais en une liberté dépassant les bornes communément admises par rapport aux affects tragiques et à la perspective morale concernant la violence. À la lumière du modèle de la catharsis, il est pourtant faux - c'était, on s'en rappelle, l'erreur de Platon - de concevoir l'allègre transgression des affects tragiques et de leur perspective morale en art de telle façon qu'elle provoque ou sinon proclame la transgression violente de la perspective morale en politique. Car à la lumière du modèle de la catharsis, on peut voir comment la libération par rapport à la perspective morale en art peut avoir un effet bénéfique pour la prise en considération de la perspective morale en politique.

La façon la plus évidente et la plus simple de déterminer cet effet bénéfique, c'est au moyen du concept de délestement. L'effet cathartique de l'art y est conçu de telle manière qu'il permet une désactivation des besoins dont la satisfaction est tout aussi nécessaire qu'elle est menaçante en dehors de l'art, soit dans la vie sociale et politique. À cause de la satisfaction, obtenue de manière substitutive en art, de ces besoins inassouvis en dehors de l'art, nous voyons ceux qui la ressentent - comme l'écrit Aristote " semblable à ces gens qui, ayant pris des remèdes et des purgatifs, retrouvent leur aplomb "(Politique, 1342 a). En appliquant ce raisonnement à la liberté esthétique par rapport à la perspective morale, cela signifie que nous avons besoin en art d'espaces de manœuvre d'une telle liberté afin-comme on le lit chez Arnold 
Gehlen - de pouvoir supporter la "pression impérieuse de la société "en dehors de l'art. Un art qui-comme le dit encore Gehlen - nous "[fait] miroiter des degrés de liberté et des éveils de la réflexion et des privautés libertines qui ne peuvent pas du tout être accommodées dans la vie publique * remplit ainsi le rôle délestant du " petit gnome démonique et affairé auquel, dans chaque maison, on doit laisser une porte ouverte ". La bonne administration du ménage - c'est ce que signifie "économie" - ne repousse pas au dehors ce qui la menace et ne l'expulse pas comme Platon voulait le faire avec les poètes, mais l'intègre : en tant que ce qui procure le délestement.

Si convaincante que puisse sembler cette explication de l'effet cathartique de l'art pour la politique, elle a pourtant besoin d'être complètée. Car si on a maintenant, suivant la proposition de Gehlen, ouvert la porte de la maison au petit gnome démonique qui s'appelle "Art ", alors il nous arrive ce que Benjamin raconte à propos du "petit homme bossu": on le rencontre partout dans la maison, et quand on le rencontre, sous le charme de son regard étrange, ce qui est à soi se brise, se réduit de moitié, éclate : "Lorsqu'il apparaissait, j'avais le dessous. Ayant le dessous, j'avais un regard auquel les choses se dérobaient, jusqu'à ce que le jardin, au bout d'une année, soit devenu un jardinet, ma chambre une chambrette, le banc un tabouret. Elles rapetissaient, et c'était comme si une bosse leur poussait, qui s'incorporait dès lors elle-même au monde du petit homme pour très longtemps. Le petit homme m'avait précédé partout là où j'allais ${ }^{10}$ ". L'art, même dans une économie de la différence, n'est pas confiné à un lieu fixe et retiré où il nous promettrait un congé des fatigues du politique et du social et de leur "pression "morale (Gehlen). La liberté de l'art par rapport à la morale quitte plutôt à chaque fois le lieu auquel nous tentons de le confiner et suspend la vie politique et sociale au milieu de sa course. La différence entre art et politique n'est pas pour autant suspendue : le fait que le gnome démonique peut apparaitre dans la maison en tout temps et en tout lieu ne le rend pas moins étranger aux choses et à l'ordre de la maison. Mais cela montre que l'économie de cette différence ne peut s'en remettre à des délimitations de domaines bien établies. Non seulement l'art et sa liberté par rapport à la morale nous distraient de la politique et de sa responsabilité vis-à-vis de la morale, mais il la menace aussi ; non pas certes comme la logique de l'identité nous présente cette menace, soit celle de sa dissolution, mais par son interruption.

9. A. Gehlen, Zeit-Bulder. Zur Soziologie und Ästhetik der modemen Malerei, Athenäum, Francfort/Bonn, 1965, p. 222 sqq. Pour un commentaire plus détaillé, voir Ch. Menke, Die Souveränität der Kunst. Ästhetische Erfahring nach Adomo und Derrida, Suhrkamp. Francfort, 1991, p. 200 sqq. La Souveraineté de l'art L'Expérience esthétique après Adorno et Derrida trad. fr. P. Rusch, “ thêories ", Paris, A. Colin, 1993, p. 200 sqq.

10. W. Benjamin, Berliner Kindheit um Neunzehnhundert, Suhrkamp, Francfort 1975, p. 164 sqq. ("Enfance berlinoise vers l'an mil neuf cent", trad. J. Lacoste dans Sens unique, Paris, Éd. M. Nadeau, 1978, 1988). Sur l'ambivalence de l'art évoquée plus loin, qui comme purification est en même temps une menace, comme remède aussi un poison, voir la discussion chez J. Derrida, "La pharmacie de Platon ", La dissémination, Seuil, Paris 1972, p. 69-196. 
Or, même cette menace en tant qu'envers de la médaille du délestement - soit la menace que présente l'art encore aussi dans le cadre d'une économie de la différence - remplit aussi une fonction bénéfique pour la politique. Cette fonction bénéfique de l'art se laisse aussi comprendre comme effet cathartique ou "purificateur". Mais tandis que dans la compréhension de la catharsis dans le concept gehlenien du délestement il était présupposé que par là rien ne changeait $a ̀$ et dans la politique, l'effet cathartique de la menace esthétique consiste justement en un changement dans la compréhension du politique et dans sa façon de procéder. La catharsis esthétique redevient ainsi ce qu'elle ètait déjà aussi chez Aristote $^{11}$ : à savoir une connaissance critique. L'interruption par l'art purifie le politique et le social des orientations mal choisies et des modèles faux. Il s'ensuit que l'effet positif de la liberté par rapport à la morale en art pour la responsabilité envers la morale en politique et dans la société consiste en la remise en question radicale de la forme établie et de l'ordre admis de cette responsabilité : en une désautorisation qui l'interrompt. C'est en cela que consiste la nécessité politique d'un art moderne de la liberté, malgré tout son immoralisme. Si l'on ouvre une porte au gnome démonique, ou plus précisément: le gnome démonique de l'art s'ouvre une porte (car comment pourrait-on lui barrer tout accès ?), cela n'aura pas pour effet de faire tomber la maison. Mais cela change l'ordre dans la maison - cela change notre opinion sur la constitution de cet ordre et sur la solidité qu'il peut avoir. Ce que Benjamin a écrit à propos du petit bossu s'applique donc encore pour l'effet de l'art sur l'ordre dans la politique et la socièté : "celui que le petit homme regarde n'accorde aucune attention. $\mathrm{Ni}$ à lui-mème ni au petit homme non plus. Il se tient debout, interdit, devant un tas de débris". L'effet purificateur de l'art et de sa liberté tient dans le regard brisé qu'il nous confère: un regard auquel les brisures dans les ordres sociaux et politiques se dévoilent.

Université libre de Berlin

Traduit de l'allemand par Philippe Constantineau

11. La question de savoir si la catharsis chez Aristote a de fait un sens cognitif et mème critique est, dans l'histoire de l'interprétation de la Poétique, notoirement controversée (et il est difficile de trancher la question en raison de la minceur des sources textuelles). Voir là-dessus les textes rassemblés par M. Luserke (éd.), Die aristotelische Katharsis. Dokumente ihrer Deutung in 19. und 20. Jahrhundert. Olms, Hildesheim, 1991 ; et dans ce volume en particulier K. Gründer, "Jacob Bernays und der Streit um die Katharsis * (p. 352-385). Pour la conception de la catharsis comme critique, voir aussi M. Nussbaum, The Fragility of Goodness. Luck and Ethics in Greek Tragedy and Philosophy, Cambridge University Press, Cambridge 1986, p. 388 sqq. 